\title{
Visualization of Crystallographic Defects in InSb Micropillars by Ptychographic Topography.
}

\author{
Mariana Verezhak $^{1, *}$, Steven Van Petegem ${ }^{1}$, Vincent Jacques ${ }^{2}$, Pierre Godard ${ }^{3}$, Klaus Wakonig ${ }^{1}$, \\ Ludovic Thilly ${ }^{3}$ and Ana Diaz ${ }^{1}$ \\ 1. Paul Scherrer Institute, Villigen, Switzerland. \\ 2. Laboratoire de Physique des Solides, Universite Paris-Sud, CNRS, UMR 8502, Orsay, France. \\ 3. Institut Pprime, CNRS-University of Poitiers-ENSMA, SP2MI, Futuroscope, France. \\ * Corresponding author, mariana.verezhak@psi.ch
}

Investigation of the strain field and defects in crystalline materials is essential in materials characterization, fabrication and design, as they are responsible for distinct mechanical, electric and magnetic properties of a desired material. Therefore, the visualization of strain and its relation to the type and density of defects in the crystal at the nanoscale is required. A domain in which such questions are particularly relevant is the fabrication of nanodevices for microelectronics from semiconductors, such as InSb, that are used as fast transistors, detectors and sensors. Classically, transmission electron microscopy (TEM) provides imaging of the crystalline defects with atomic spatial resolution, but due to the thin sections requirement, sample preparation is invasive and can modify the strain fields to be analyzed. A conventional tool to non-invasively study strain is Laue X-ray micro-diffraction [1], which reveals the strain field in crystalline samples averaged over the direction of the beam propagation with a resolution limited by the beam size. X-ray topography (XRT) [2] has been routinely used for imaging defects based on the diffraction contrast, with the resolution being restricted by the detector pixel size.

X-ray coherence methods, such as coherent diffraction imaging (CDI) and ptychography, which are based on measuring the sample's far-field diffraction patterns and using phase retrieval algorithms, permit obtaining high resolution images. If the measurements are performed close to a Bragg peak, the resulting image becomes highly sensitive to the presence of strain [3,4]. We have recently developed ptychographic topography, in which a crystalline sample is rotated with respect to the incident beam such that a certain atomic plane is in the Bragg condition, as shown in Fig. 1a [5]. A pinhole is then placed after the sample in the forward direction and is spatially translated, providing the sufficient overlapping necessary for ptychographic reconstructions [6]. The diffraction patterns are recorded at each pinhole position with a $2 \mathrm{D}$ detector downstream of the pinhole and used simultaneously for the reconstruction of the wave front at the pinhole position. So far measurements were performed in forward direction due to the limited space at the beamline to fulfil the ptychographic detector sampling requirement along the Bragg-diffracted beam direction. Numerical backpropagation then enables one to obtain an image of the sample, which is sensitive to the lattice displacements caused by defects.

In this work, we present the applicability of ptychographic topography to visualize crystalline defects in InSb micropillars after mechanical compression. This model system has well-understood features: it

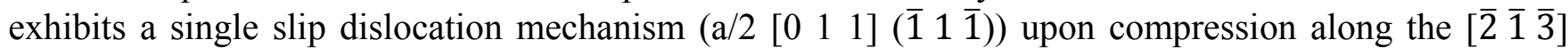
pillar axis [7]. Such partial dislocations leave extended stacking faults (SF) (a/3[ $\overline{1} 1 \overline{1}])$. However, the fact of having the tip of a micro-compression device in contact with the pillar surface may add an extra constraint for plastic deformation, which can result in additional strain caused by lattice deformation and rotation of the crystallographic planes. 
Experiments were performed at the cSAXS beamline (SLS, Switzerland) at the energy of $5.5 \mathrm{keV}$. The sample was brought to the Bragg condition of the 220 reflection, confirmed by performing a rocking curve scan with the beam focused by a Fresnel zone plate (FZP) to about $100 \mathrm{~nm}$ spot size while probing the Bragg diffracted intensity with an Eiger 500k detector. At this reflection, the scalar product of the

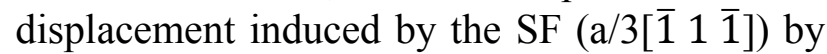
the scattering vector of the 220 Bragg reflection is zero; therefore we were not sensitive to slip planes but to other defects present in the crystal.

Once the specific sample area was close to the Bragg condition, we let a parallel beam illuminate the whole sample as show in Fig. 1a. A pinhole of $3 \mu \mathrm{m}$ in diameter was placed $3 \mathrm{~mm}$ downstream of the sample in the forward direction and scanned with a 2D piezoelectric stage such that the average step size was $1 \mu \mathrm{m}$, i.e. with sufficient overlapping for ptychography. Diffraction patterns in the forward direction were recorded at each scanning position with the Pilatus $2 \mathrm{M}$ detector $\sim 7.4 \mathrm{~m}$ downstream of the pinhole at a sample rotation of $0.08^{\circ}$ from the Bragg angle. They were then used as input in the iterative phase retrieval algorithms, allowing the reconstruction of the complex-valued wave front at the pinhole position, which could then be back propagated to the sample plane. When the sample is in Bragg condition, the amplitude image, with a resolution of $100 \mathrm{~nm}$ (estimated by Fourier shell correlation), reveals the presence of a lattice distortion close to the base of the pillar as shown in Fig. 1c with the red arrow, while out of Bragg condition such defect is not observed (Fig. 1b). This defect could originate from the experimental constraints of the micro-compression. Hence, ptychographic topography proves itself beneficial to answer specific questions concerning strain fields in crystalline materials with the perspective of application to extended specimens [8].

\section{References:}

[1] G. E. Ice \& J. W. L. Pang, Mater. Charact. 60 (2009) 1191-1201.

[2] G. N. Ramachandran, Proc. Indian Acad. Sci. A 19 (1944) 280-292.

[3] M. Pfeifer et al, Nature 442 (2006) 63-66.

[4] P. Godard et al, Nat. Commun. 2 (2011) 568.

[5] S. Van Petegem et al, (2018) in prep.

[6] E. H. R. Tsai et al, Opt. Express 24 (2016) 6441.

[7] L. Thilly et al, Philos. Mag. 92 (2012) 3315-3325.

[8] This project has received funding from the European Union's Horizon 2020 research and innovation program under the Marie Skłodowska-Curie grant agreement No 701647", SNF grant No 200021L_169753, ANR-16-CE93-0006, by "Investissement d'Avenir" (LABEX INTERACTIFS, ANR-11-LABX-0017-01) and by Nouvelle Aquitaine Region / European Structural and Investment Funds (ERDF No P-2016-BAFE-94/95). 